Review

\title{
Zika virus: History, emergence, biology, and prospects for control
}

\author{
Scott C. Weaver a, b, *, Federico Costa ${ }^{\text {c, d }}$, Mariano A. Garcia-Blanco ${ }^{\text {e, f }}$, Albert I. Ko ${ }^{\text {c, g, }}$

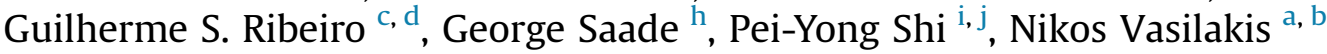

\footnotetext{
${ }^{a}$ Institute for Human Infections and Immunity, Department of Microbiology \& Immunology, University of Texas Medical Branch, Galveston TX, USA

${ }^{\mathrm{b}}$ Institute for Human Infections and Immunity, Department of Pathology, University of Texas Medical Branch, Galveston TX, USA

c Centro de Pesquisas Gonçalo Moniz, Fundação Oswaldo Cruz, Ministério da Saúde, Salvador, BA, Brazil

d Instituto da Saúde Coletiva, Universidade Federal da Bahia, Salvador, BA, Brazil

e Department of Biochemistry \& Molecular Biology, University of Texas Medical Branch, Galveston, TX, USA

${ }^{\mathrm{f}}$ Programme in Emerging Infectious Diseases, Duke-NUS Medical School, Singapore

${ }^{g}$ Department of Epidemiology of Microbial Diseases, Yale School of Public Health, New Haven, CT, USA

${ }^{\mathrm{h}}$ Department of Obstetrics and Gynecology, University of Texas Medical Branch, Galveston, TX, USA

i Department of Biochemistry \& Molecular Biology, and Sealy Center for Structural Biology \& Molecular Biophysics, University of Texas Medical Branch, Galveston, TX, USA

j Department of Pharmacology \& Toxicology, and Sealy Center for Structural Biology \& Molecular Biophysics, University of Texas Medical Branch, Galveston,
} TX, USA

\section{A R T I C L E I N F O}

\section{Article history:}

Received 29 February 2016

Received in revised form

9 March 2016

Accepted 12 March 2016

Available online 18 March 2016

\begin{abstract}
A B S T R A C $T$
Zika virus (ZIKV), a previously obscure flavivirus closely related to dengue, West Nile, Japanese encephalitis and yellow fever viruses, has emerged explosively since 2007 to cause a series of epidemics in Micronesia, the South Pacific, and most recently the Americas. After its putative evolution in sub-Saharan Africa, ZIKV spread in the distant past to Asia and has probably emerged on multiple occasions into urban transmission cycles involving Aedes (Stegomyia) spp. mosquitoes and human amplification hosts, accompanied by a relatively mild dengue-like illness. The unprecedented numbers of people infected during recent outbreaks in the South Pacific and the Americas may have resulted in enough ZIKV infections to notice relatively rare congenital microcephaly and Guillain-Barré syndromes. Another hypothesis is that phenotypic changes in Asian lineage ZIKV strains led to these disease outcomes. Here, we review potential strategies to control the ongoing outbreak through vector-centric approaches as well as the prospects for the development of vaccines and therapeutics.
\end{abstract}

(c) 2016 Elsevier B.V. All rights reserved.

\section{Contents}

1. History of Zika virus discovery and early characterizations of distribution, transmission cycles and disease ................... 70

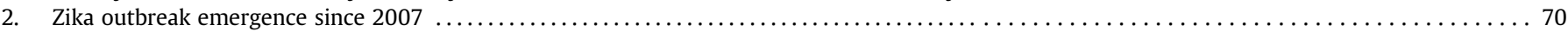

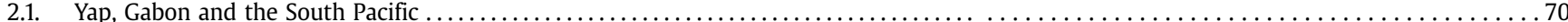

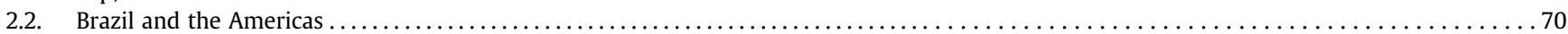

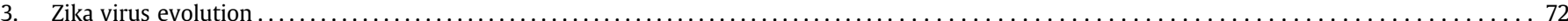

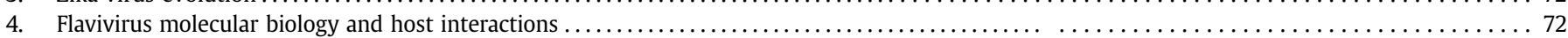

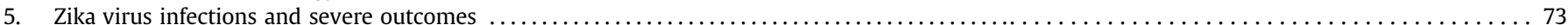

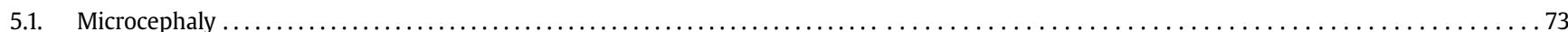

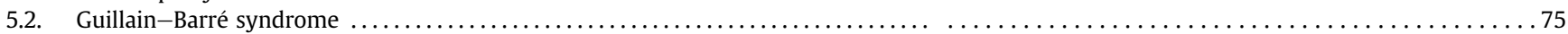

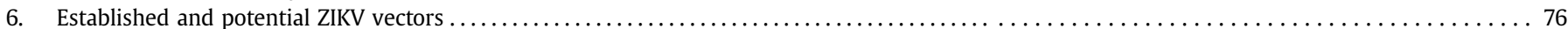

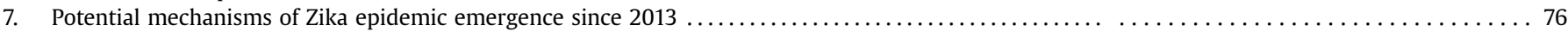

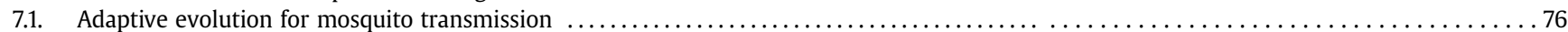

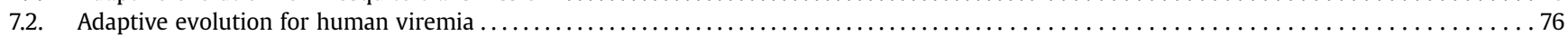

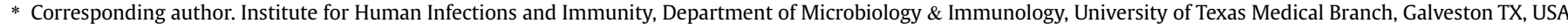
E-mail address: sweaver@utmb.edu (S.C. Weaver).
} 


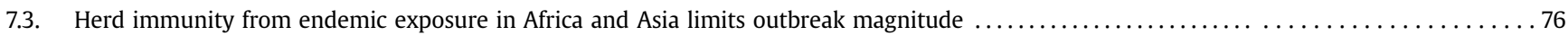

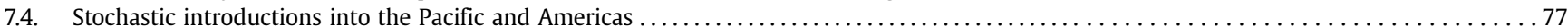

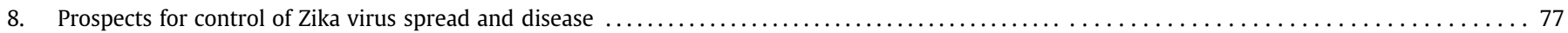

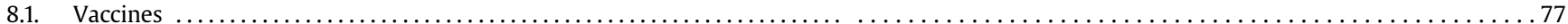

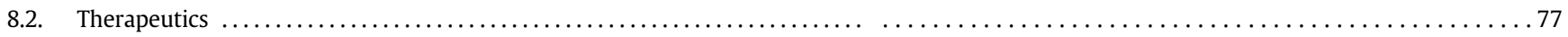

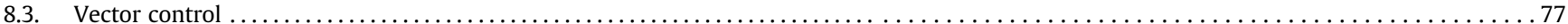

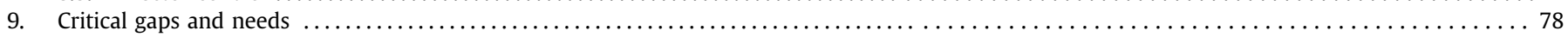

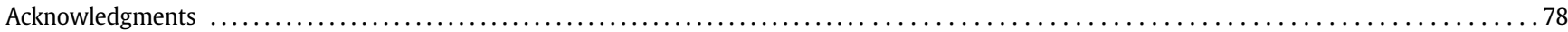

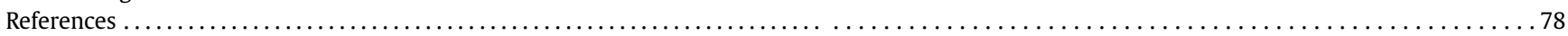

\section{History of Zika virus discovery and early characterizations of distribution, transmission cycles and disease}

Zika virus (ZIKV) was discovered in the Zika Forest of Uganda during research supported by the Rockefeller Foundation to study the enzootic or sylvatic cycle of yellow fever virus and to identify additional arboviruses. Sentinel Rhesus monkeys were placed into the canopy in April, 1947 and one developed fever and viremia, leading to ZIKV isolation by inoculation of its blood intracranially into infant mice. The following January, ZIKV was isolated from the canopy-dwelling mosquito Aedes africanus collected at the same site (Dick et al., 1952). The first characterization of human disease following ZIKV infection (2 cases based on seroconversion and one case based on virus isolation from the serum of a febrile girl) occurred in Nigeria during 1954 (Macnamara, 1954). These cases were accompanied by fever, headache, diffuse joint pain, and in one case, slight jaundice. Two years later, experimental infection of a yellow fever-vaccinated human volunteer with a 6th mouse brain-passaged ZIKV strain from the 1954 Nigerian human isolates resulted in a relatively mild febrile illness (slight headache, malaise, fever) associated with natural infection. Attempts to demonstrate transmission by Aedes (Stegomyia) aegypti mosquitoes fed on the infected volunteer did not succeed (Bearcroft, 1956). The first direct detection of ZIKV in Asia as well as the first evidence of transmission by an urban vector occurred when the virus was isolated from $A$. aegypti mosquitoes collected in Malaysia in 1966 (Marchette et al., 1969). Eleven years later, the first human infections in Asia were diagnosed from central Java in Indonesia by seroconversion in seven patients presented with fever, malaise, stomach ache, anorexia and dizziness (Olson et al., 1981).

In addition to direct evidence of the range of ZIKV circulation from these isolations and patient diagnoses, human serosurveys have suggested a broad range in both Africa and Asia (Fig. 1). However, it must be noted that several of these studies used relatively nonspecific methods such as hemagglutination inhibition that show extensive cross reactions among flaviviruses, and some did not test for seropositivity for other flaviviruses now known to circulate in these regions. Regardless, the combined evidence of virus and antibody detection strongly suggests widespread circulation in both continents. Whether Asia, like Africa, is the home to enzootic foci of circulation is unknown and will be difficult to determine unless remote forests far from potential humanmosquito circulation can be studied or evolutionarily independent ZIKV lineages can be associated with enzootic circulation, as has been documented for dengue viruses (Wang et al., 2000). Prior to 2014, there was no evidence of ZIKV circulation in the Western Hemisphere.

\section{Zika outbreak emergence since 2007}

\subsection{Yap, Gabon and the South Pacific}

The first significant outbreaks of ZIKV infection involving more than a few persons were detected in 2007. Following outbreaks tentatively attributed to dengue and chikungunya viruses, 4312 sera from patients presenting with painful febrile disease in Libreville, Gabon and towns to the north, and 4665 mosquitoes collected in the same regions, were retrospectively screened by RTPCR for ZIKV. Five human sera and two Aedes albopictus pools, all sampled in an urban locations, tested positive (Grard et al., 2014). Also in 2007, Yap, an island that is part of the Federated States of Micronesia, experienced an outbreak of ZIKV infection accompanied by fever, rash, conjunctivitis, and arthralgia (Duffy et al., 2009) (Fig. 1). A total of 49 confirmed and 59 probable cases were diagnosed using a combination of RT-PCR and serological analyses, with the prior presence of dengue virus resulting in some uncertainty in the latter test interpretations. Up to 73\% of the 7391 Yap residents were estimated to have been infected and Aedes (Stegomyia) hensilli was identified retrospectively as the likely principal vector.

Next, in 2013, ZIKV reached French Polynesia, probably introduced from somewhere in the Southeast Asia area rather than from Yap (see below), followed by spread to several other islands in Oceania including New Caledonia, the Cook Islands, and Easter Island (Fig. 1). A. aegypti and A. albopictus are found throughout most of this region (Horwood et al., 2013). The French Polynesian outbreak was estimated to have affected approximately 28,000 persons or approximately $11 \%$ of the population, who sought medical care based on typical signs and symptoms of low-grade fever, maculopapular rash, arthralgia, and conjunctivitis (Musso et al., 2014). Coincidentally, during this outbreak, Guillain-Barré syndrome (GBS) was for the first time associated with ZIKV. Also during this outbreak, the first evidence of risk for transmission through blood banks, and detection of ZIKV (or viral RNA) in semen, saliva and urine were documented. Mosquito-borne transmission in French Polynesia may have involved Aedes (Stegomyia) polynesiensis.

\subsection{Brazil and the Americas}

Late in 2014, clusters of patients presenting with rash, mild fever, and arthralgia were first noticed in some municipalities of Northeastern Brazil. Case numbers increased during the first months of 2015, and the illness rapidly spread throughout the region. In April 29, 2015, researchers from Salvador, the largest state capital in the Northeast of Brazil, announced ZIKV as the likely etiology for the outbreak (Campos et al., 2015). Applying RT-PCR to serum samples from 24 patients presenting with an acute nonspecific illness in Camaçari, a city in metropolitan Salvador, 


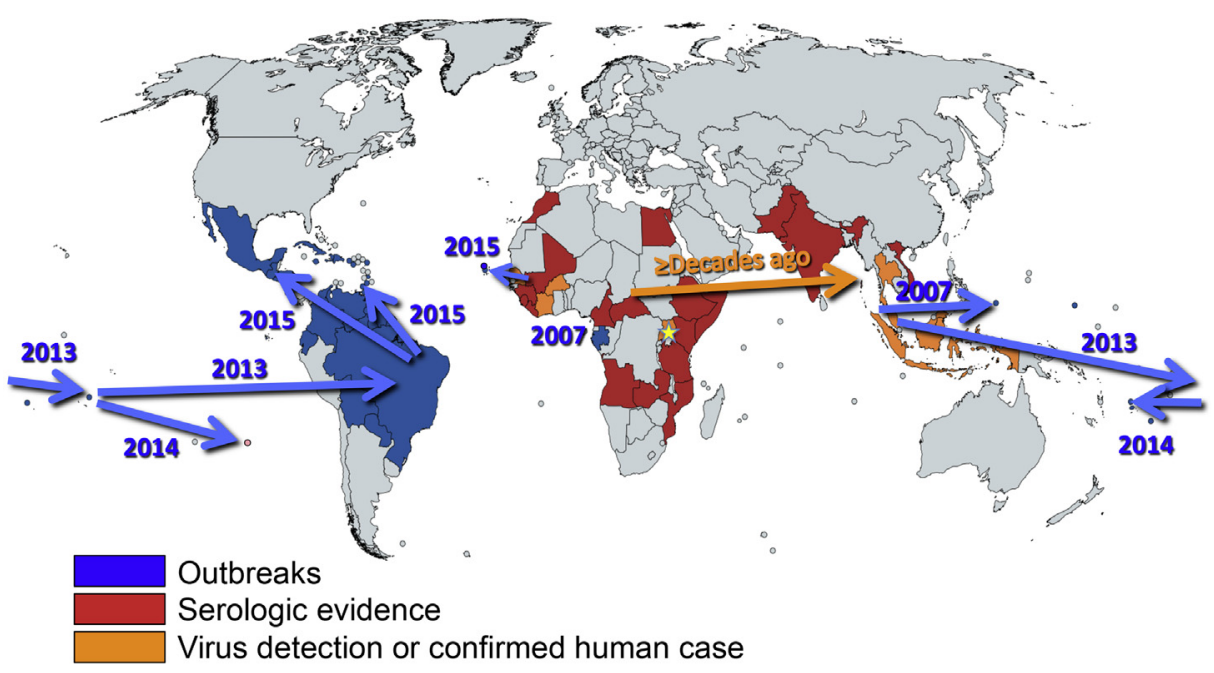

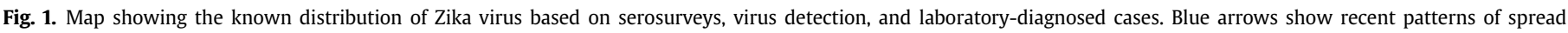
deduced from phylogenetic studies (see Fig. 1). The yellow star shows the location of the Zika forest where the virus was discovered in 1947.

they confirmed the diagnosis of ZIKV infection in seven of them. Additionally, they found that another three patients were RT-PCR positive for CHIKV. Almost simultaneously, ZIKV was also confirmed by RT-PCR in 8 of 21 patients exhibiting a dengue-like illness from Natal, another large city in Northeast Brazil (Zanluca et al., 2015).

Two main hypotheses were raised to explain how ZIKV entered Brazil. The first is that the virus was introduced during the World Cup soccer games, held in the country between June and July of 2014 (Zanluca et al., 2015). However, because no ZIKV-endemic Pacific countries participated in the tournament, a second hypothesis was raised, suggesting that the virus entered Brazil during the 2014 World Sprint Championships, a canoeing race held in Rio de Janeiro in August, 2014 with the participation of athletes from four Pacific countries where ZIKV circulated in 2014 (French Polynesia, New Caledonia, Cook Islands, and Easter Island) participating (Musso, 2015). Based on the initial propagation of ZIKV in the Northeast of Brazil, we think that it is most likely that the virus entered through Natal, Recife, Salvador and/or Fortaleza, which held the soccer games during the 2014 World Cup. However, recent phylogenetic studies using coalescent methods suggest the introduction occurred between May and December, 2013 (Faria et al., 2016). Regardless of its initial port of entry into Brazil, ZIKV rapidly reached the entire country and, by the end of 2015 , laboratory-confirmed autochthonous cases had been identified in all five regions. Probably from Brazil ZIKV continued to spread to other countries in the American continent. As of, autochthonous cases had been diagnosed in 33 countries and territories in the Americas (http://ais.paho.org/phip/viz/ed_zika_countrymap.asp).

Accurate data on ZIKV incidence in Brazil and in the Americas is not available, as until recently Zika was not subjected to mandatory reporting. To date, the most comprehensive epidemiological investigation in the region was performed in Salvador, Brazil, where the Epidemiological Surveillance Office established 10 public emergency health centers as sentinel units for surveillance of patients with acute exanthematous illness of unknown cause during 2015. Between February 15 and June 25, this surveillance identified 14,835 indeterminate acute exanthematous illness cases; an attack rate of 5.5 cases per 1000 inhabitants. Among 58 patient sera tested by RT-PCR, ZIKV was confirmed in 3 (5.2\%), CHIKV in 3 (5.2\%), DENV-3 in 1 (1.7\%), and DENV-4 in 1 (1.7\%), indicating that these arboviruses were co-circulating during the outbreak. Based on the clinical and epidemiological characteristics of the cases, ZIKV was assumed as the most common etiology for the outbreak.

The Brazilian Ministry of Health estimates that between 497,593 and 1,482,701 ZIKV infections have occurred in the country since the epidemics began (http://portalsaude.saude.gov.br/images/pdf/ 2016/janeiro/22/microcefalia-protocolo-de-vigilancia-e-respostav1-3-22jan2016.pdf). In Colombia, another South American Country greatly affected by ZIKV since late $2015,31,555$ cases have been reported, 1504 of them laboratory confirmed (WHO. Situation Report. 19 Feb 2016; http://apps.who.int/iris/bitstream/10665/ 204454/1/zikasitrep_19Feb2016_eng.pdf?ua=1).

The burden of ZIKV-associated complications is rapidly increasing in Brazil and in other countries. Like in French Polynesia (Musso et al., 2014), an increase of GBS cases was reported during the ZIKV outbreaks in the Northeast of Brazil, Colombia, El Salvador, Suriname and Venezuela (WHO. Situation Report. 19 Feb 2016; http://apps.who.int/iris/bitstream/10665/204454/1/zikasitrep_ 19Feb2016_eng.pdf?ua=1). However it was the unusual rise in the number of newborns with microcephaly (MC) that began months after cases rose in northeastern Brazil, that was the trigger for international alarm including the recent WHO declaration of Public Health Emergency of International Concern WHO statement on the first meeting of the International Health Regulations (2005) (IHR 2005) Emergency Committee on Zika virus and observed increase in neurological disorders and neonatal malformations. Feb 1, 2016; http://www.who.int/mediacentre/news/statements/2016/1stemergency-committee-zika/en/ (accessed Feb 3, 2016). Since the beginning of the microcephaly outbreak until February 13th 2016, more than 5200 cases have been notified by the Brazilian Ministry of Health (http://portalsaude.saude.gov.br/images/pdf/2016/ fevereiro/17/coes-microcefalia-inf-epi-13-se06-2016.pdf), but only a fraction $(\sim 37 \%)$ of them were associated to congenital ZIKV infection. This MC is causing a catastrophic impact on the socioeconomic status of affected families. Mothers of newborns with MC usually need to leave work in order to devote full-time care to those children with severely compromised cognitive and motor skills.

The causal relation between Zika infection during pregnancy and MC in newborns has not been absolutely established. Three main lines of evidence support this link: 1) as described above, 
there was a higher incidence of $\mathrm{MC}$ in newborns from regions where a previous outbreak of ZIKV was described; 2) virus has been detected in the amniotic fluid and tissues of $>10$ fetuses and newborns with microcephaly (Calvet et al., 2016; Mlakar et al., 2016) (Martines et al., 2016; Sarno et al., 2016) from different parts of Brazil; and 3) A high proportion of the fetuses of women who were documented to have symptomatic Zika virus infection during pregnancy subsequently developed defects, including microcephaly during gestation (Brasil et al., 2016). In a recently published study, among a cohort of 88 women presenting with an acute episode of rash during pregnancy, ZIKV was found to be the cause in 72 (82\%) by RT-PCR testing (Brasil et al., 2016). During follow-up of 42 women who developed symptomatic ZIKV infection during pregnancy, fetal abnormalities were identified in $29 \%$. In Brazil, mothers giving birth to newborns with MC have reported fever, rash and conjunctivitis, the most commons signs and symptoms of ZIKV infection, during pregnancy. However, diagnosis beyond the earliest stage of acute disease is nearly impossible in the dengue-endemic regions where ZIKV is circulating. Furthermore, the majority of exposures to ZIKV may cause an asymptomatic infection in this population, so the large majority of infected pregnant women may remain undiagnosed (Sarno et al., 2016). To date, it remains unknown if the rate of fetal abnormalities in women who develop asymptomatic infection will be as high as that observed in pregnant women who develop symptomatic disease.

Controlling Zika epidemics in Brazil and other settings is a major challenge, as the cornerstone is for success interrupting the transmission cycle. Recent attempts to control dengue, which shares a similar or identical urban transmission cycle, by relying on A. aegypti control has largely failed. However, it is not clear if other species of mosquitos significant ZIKV vectors in Brazil. The Brazilian Ministry of Health recommends the use of repellents; however there is no evidence that this method reduces contact with mosquitoes enough to prevent ZIKV infection and transmission. The potential for prolonged presence of ZIKV in semen (Atkinson et al., 2016) and the few cases suggesting sexual transmission outside of Brazil, indicate the potential existence of alternative routes of human-human transmission (Costa et al., 2016).

\section{Zika virus evolution}

ZIKV, like dengue viruses (DENV) may be restricted in it natural vertebrate host range, generally including primates as its amplification and reservoir hosts. Thus, the evolution of ZIKV has been associated mostly by sampling of strains from the enzootic cycle in Africa, its rapid expansion in the first 2 decades of the 21 st Century in Asia and Oceania, and more recently by its introduction into the Americas (Figs. 1 and 2). As has been seen observed with DENV (Chen and Vasilakis, 2011), ZIKV evolution is fueled by the global spread of the anthropophilic vector $A$. aegypti, the increase of the urban human population size, and expansion of international commerce and travel. While the prototype ZIKV strain was isolated in 1947, previous analyses based on partial envelope and NS5 gene sequences suggest that the ancestor of known ZIKV strains occurred sometime in the early 1900s in Uganda (Faye et al., 2014). ZIKV has spread throughout sub-Saharan Africa, westwards to Nigeria and Senegal as well as eastwards to Eastern Africa and Asia (likely hundreds of years ago). Our current analyses based on complete genome sequences reiterate the notion that ZIKV likely originated in Africa and diverged into two major lineages: African and Asian/American (Figs. 3, 4). The African strains fall into two distinct groups. The first group (Uganda cluster) is anchored by the prototype MR766 strain (Dick et al., 1952) and includes isolates from Senegal and Central African Republic from 1947 to 2001; the second group (Nigeria cluster) includes strains isolated in Nigeria and Senegal from 1968 to 1997 . The tree topology suggests that at least two distinct lineages are circulating in Senegal, supporting the notion of multiple introductions over a period of time. Most of the African lineage strains were isolated from enzootic vectors (Diallo et al., 2014; Faye et al., 2014), reflecting continuous surveillance efforts in Senegal. The Asian cluster is anchored by the prototype P6-740 strain isolated in Malaysia in 1966 (Marchette et al., 1969) and includes strains isolated in Cambodia (Heang et al., 2012), Micronesia (Lanciotti et al., 2008) and French Polynesia (Oehler et al., 2014), suggesting expansion of the Asian lineage throughout Southeast Asia. Within this cluster, a new lineage (American) emerged with the introduction of the virus into the Western Hemisphere, and now includes strains from Brazil (Mlakar et al., 2016), Puerto Rico (Thomas et al., 2016), Haiti, Guatemala, and Suriname (Enfissi et al., 2016). A major characteristic of the American ZIKV lineage is its rapid radiation, consistent with a pattern of intense diversification, as the lineage expands into new territories with immunologically naïve populations.

A recent report suggested of ZIKV recombination in field isolates (Faye et al., 2014). Evidence for recombination among members of the genus Flavivirus has been reported mainly in DENV [reviewed in (Chen and Vasilakis, 2011)]. However since recombination has not been achieved experimentally despite concerted efforts (McGee et al., 2011; Taucher et al., 2010), caution should be exercised when inferring conclusions about these putative recombination events. For natural recombination leading to the transmission of a recombinant strain to be conclusively confirmed, the following prerequisites should be met: (i) the recombinant crossover should be demonstrated in a single PCR amplicon following cloning to ensure it occurs in a single DNA molecule; (ii) the recombination should be demonstrated repeatedly in clonal populations of viable virus (e.g. a plaque harvest or limited endpoint dilution); and (iii) the recombinant should maintain adequate sequence conservation during post-recombination evolution.

\section{Flavivirus molecular biology and host interactions}

As ZIKV evolves it will create new molecular relationships with factors of the mosquito vector and/or the human host. These, so called host factors, can be cataloged as pro-viral (dependency factors) or anti-viral (restriction factors) and the integration of their interactions with viral protein and RNAs determines the efficiency of infection, pathogenicity, transmission, and epidemic potential.

Unfortunately, little is know about ZIKV host factors and indeed it is essential that we quickly learn about them and how they may vary among cell types (e.g., cells of the genitourinary tract or the developing nervous system) and also between individuals of different ancestries. One recent study indicated that ZIKV can infect several cell types in the skin and, like many flaviviruses, ZIKV uses multiple 'receptors' to mediate attachment and entry (Hamel et al., 2015). The authors suggest that autophagy is also proviral and that ZIKV is sensitive to treatment with Type I and Type II interferons. Another study determined that ZIKV can infect human neural progenitors derived in vitro by inducing pluripotent stem cells (hiPSCs), which suggests the virus could have the capacity to infect neuroblasts in vivo (Tang et al., 2016).

Despite the limited information on ZIKV, we can extrapolate using our knowledge of other flaviviruses and the host factors that impact their replication. Recently, several en masse methods have greatly expanded the lists of important factors. For instance genome-scale loss of function screens using RNA interference and, more recently, CRISPR/Cas gene, editing have provided the field with a list of dependency factors that are likely required for efficient replication of all members of the genus (Krishnan et al., 2008; Le Sommer et al., 2012; Ma et al., 2015; Sessions et al., 2009). 


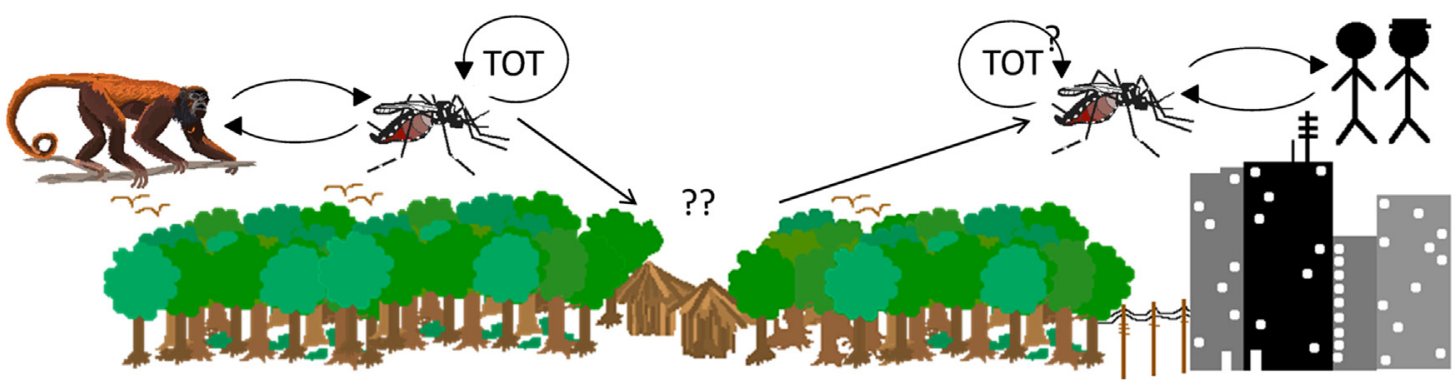

Sylvatic

\author{
Ae. africanus (Africa)* \\ Ae. dalzieli (Africa) \\ Ae. furcifer (Africa)* \\ Ae. luteochephalus (Africa)* \\ Ae. vittatus (Africa) \\ Ae. apicoargenteus (Africa) \\ Ae. hirsitus (Africa)
}

Rhesus spp (Africa)
Chlorocebus sabaeus (Africa)
Cercopithecus spp (Africa)
Colobus guereza (Africa)
Erythrocebus patas (Africa)
Pongo borneo (SE Asia) ??

\author{
Ae. metallicus (Africa) \\ Ae. opok (Africa) \\ Ae. taylori (Africa)* \\ Ae. unilineatus (Africa) \\ Ma. uniformis (Africa) \\ An. coustani (Africa) \\ Cx. perfuscus (Africa) \\ Ae. vittatus (Africa)
}

Zone of emergence
Urban

\author{
Ae. aegypti aegypti (global) \\ Ae. albopictus (global?) \\ Ae. polynensiensis (Polynesia) \\ Ae. hensilii (Polynesia) \\ Homo sapiens
}

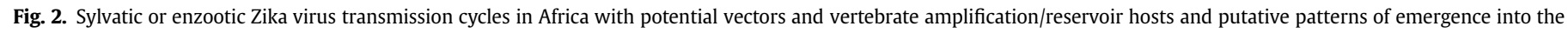
urban human-mosquito-human transmission cycle. References for vector implications are found in the text in Section 6.

Examples of dependency factors that appear to have a pan-flaviviral requirement are isoforms of the vacuolar ATPase (vATPase) and proteins required for translation on the endoplasmic reticulum, which remarkably are not required for viability of all cells. Studies are under way to test the most potent DENV and YFV host factors for an effect on ZIKV and it should be a matter of weeks before important ZIKV dependency factors are added to the current list.

A second general approach that has identified many flavivirus dependency and restriction factors is affinity chromatography coupled to quantitative mass spectrometry, where host interactors involved in lipid and RNA metabolism have emerged overrepresented (Barrows et al., 2014). In fact these biochemical methods have yielded a wealth of restriction factors. Among restriction factors there is especially an overrepresentation of RNA binding proteins, which is likely due to the fact that many of these have been involved in 'self vs. non self recognition since early in evolution (Garcia-Blanco, 2015). These restriction factors and the viral countermeasures deployed to stop them can have a strong effect on the outcome of flavivirus infection. Several laboratories are identifying the protein-protein and RNA-protein interactomes for ZIKV in both human and Aedes cells.

It is interesting that in a recent report Mlakar et al., (Mlakar et al., 2016) noted that a full-length ZIKV genome traced to the Brazil outbreak and amplified from fetal tissues had five nonsynonymous polymorphisms within the ORF when compared with French Polynesian isolate. Remarkably, three amino acid changes were found in NS1, which in DENV is secreted and has been implicated in immune evasion, one in NS4B, which can inhibit type I interferon signaling, and one in an NS5 domain that is homologous to the bacterial [ribose-2'-O]-MTase, FtsJ/RrmJ, an activity which in West Nile virus has been shown to mask the viral RNAs from host recognition (Daffis et al., 2010). This may represent a coincidence but could also be that, as expected, sequences involved in anti-host countermeasures will evolve faster than others in the flavivirus genome.

Indeed one of the most powerful anti-innate immune molecules in flaviviruses is the non-coding sfRNA (Bidet and Garcia-Blanco, 2014). In fact variation in the subgenomic flavivirus RNA (sfRNA) has been shown to lead to DENV-2 genotypes that more effectively suppress the interferon response, which likely leads to changes in epidemic potential (Manokaran et al., 2015). We have begun the analysis of the sequence variations in the ZIKV sfRNA as the virus has evolved in the past 10 years and find some intriguing differences accruing in critical RNA structures. It must be pointed out that the sfRNA can counter innate immune mechanisms in both the human host and the Aedes vector (Bidet and Garcia-Blanco, 2014).

\section{Zika virus infections and severe outcomes}

\subsection{Microcephaly}

Microcephaly refers to a head that is smaller than expected. The size of the head is typically defined by the occipito-frontal head circumference (HC), which can be measured in the fetus by ultrasound or in the neonate using a tape. The HC is then compared to a nomogram that takes into consideration the age of the fetus or neonate. Some nomograms also take into consideration gender and/or race/ethnicity. The result of the comparison is typically reported as a percentile for gestational age, or a number of standard deviations below the mean (also known as $\mathrm{Z}$ score). There is no universally-accepted nomogram or measurement cutoff used to define microcephaly. In obstetrical practice, ultrasound measurements for various fetal structures between the 10\%ile and 90\%ile are usually considered within the normal range. However, using the 


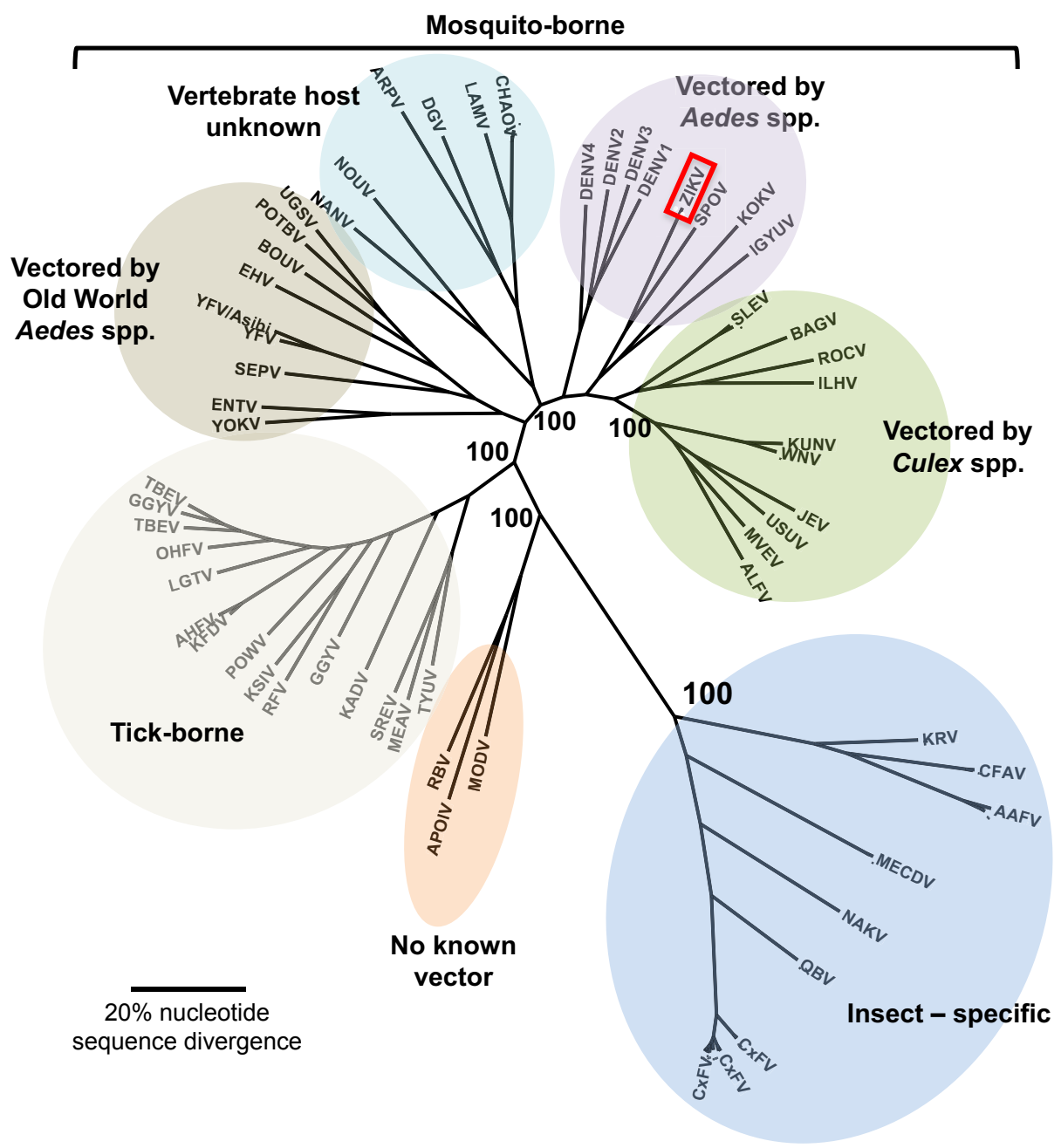

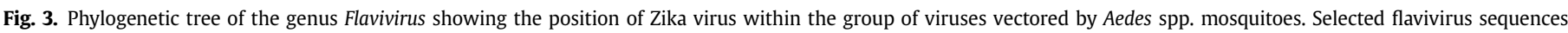

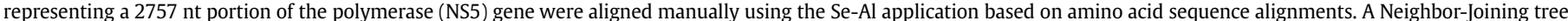

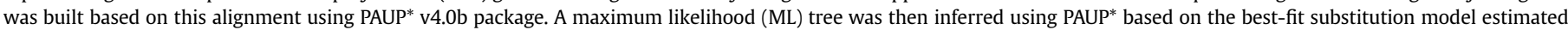

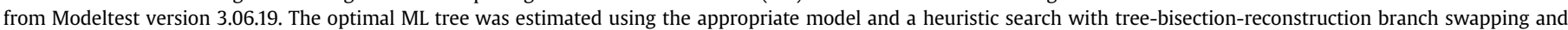

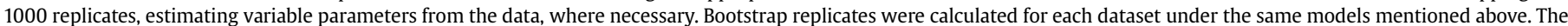
scale shows $20 \%$ nucleotide sequence divergence.

$10 \%$ ile as the cutoff for head measurement would result in a large proportion of normal fetuses being considered microcephalic (false positives). On the other hand, using a stricter cutoff may result in some false negatives. Where the cutoff is set will therefore determine the sensitivity and specificity of ultrasound in detecting microcephaly before birth. One also has to keep in mind that the fetal measurements on ultrasound have inherent errors because they rely on obtaining a cross sectional image of the head and placing calipers on the image in order to obtain the HC, rather than what happens after birth when the HC is measured directly.

In response to the concerns regarding Zika virus infection during pregnancy, the Society for Maternal Fetal Medicine (SMFM) has published a statement about ultrasound screening for fetal microcephaly following exposure. The SMFM recommends using standard deviations (SD) below the mean for gestational age to determine how to interpret the $\mathrm{HC}$ measurement and how to follow the pregnant woman (Committee, 2016). If the HC is $>2$ SDs below the mean (typically equivalent to $3 \%$ ile on most nomograms), SMFM recommends that a detailed neurosonographic examination be performed looking for periventricular and intraparenchymal echogenic foci, ventriculomegaly, cerebellar hypoplasia, and cortical abnormalities. If the anatomy is normal, then a follow up ultrasound is recommended in 3-4 weeks. The SMFM recommends that isolated fetal microcephaly be defined as a fetal HC 3SDs or more below the mean for gestational age, and that the diagnosis be considered certain if the HC is 5SDs or more below the mean. The SMFM also provides a table for the cutoffs that is based on studies in the 1980s that correlated HC measurements with adverse postnatal outcomes. This table, however, does not include values below 20 weeks of gestation. Because of this limitation, and because most ultrasound packages automatically provide the percentile for the $\mathrm{HC}$ measurement rather than SD below the mean, the 3\%ile cutoff for gestational age from the recent national cohort of normal pregnancies has been used (Marrs et al., 2016). In addition, percentile cutoffs are provided for different racial/ethnic categories. Using these cutoffs derived from a more recent and representative cohort may allow for some refinement of the sensitivity and specificity.

Regardless of what method is used to screen for or diagnose microcephaly, one has to keep in mind that it may be due to a variety of developmental factors, including genetic, infectious, and environmental exposures (Passemard et al., 2013). Therefore a 


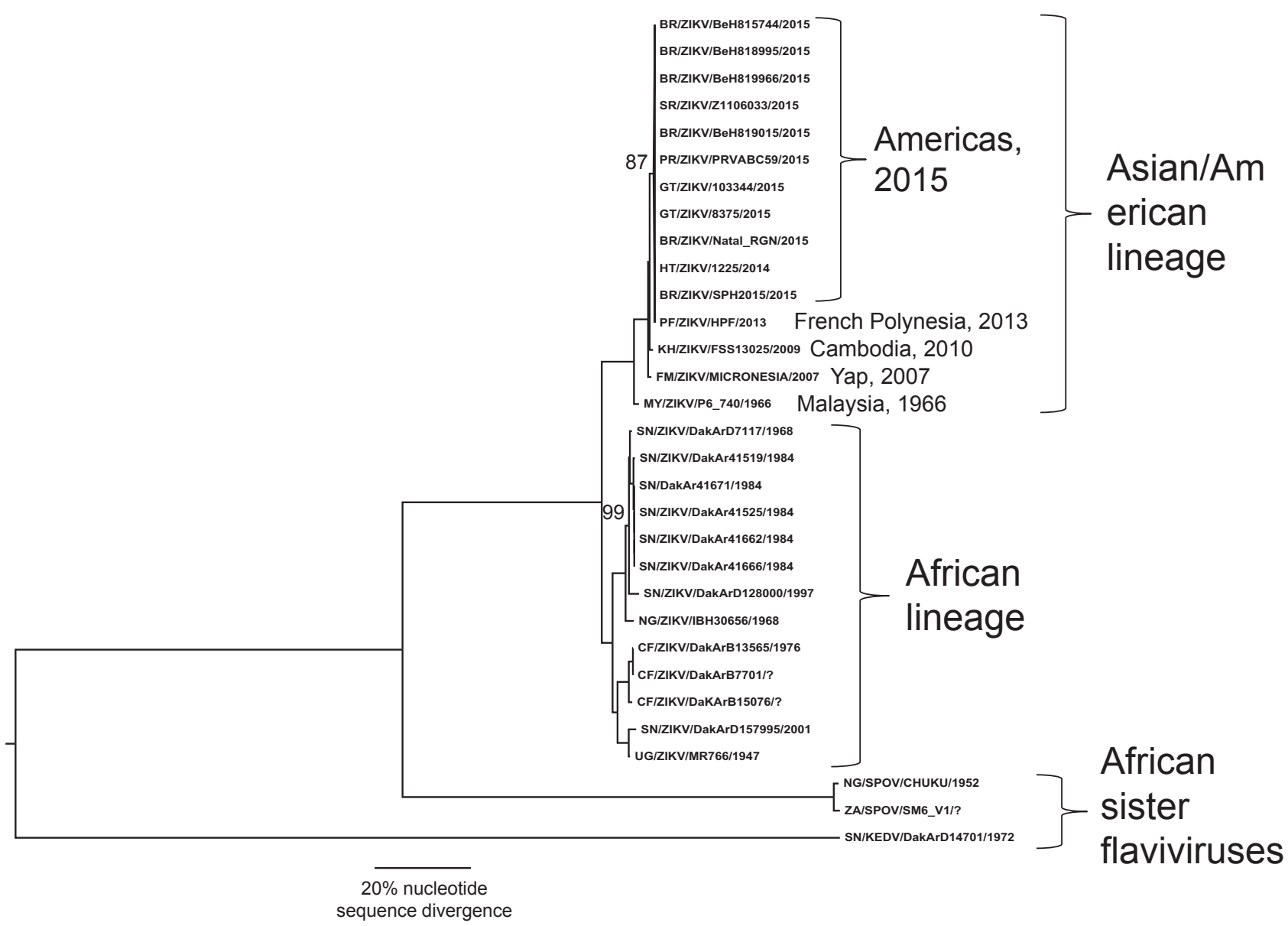

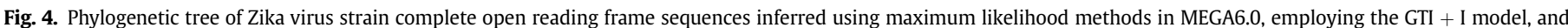

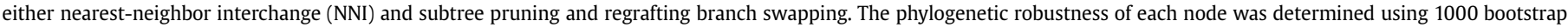

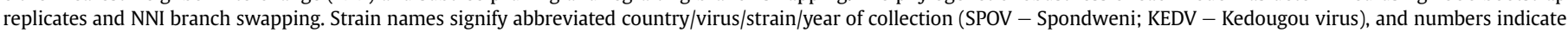
bootstrap values < $100 \%$ and scale shows $20 \%$ nucleotide sequence divergence.

workup to exclude these other conditions may be necessary. The development of microcephaly depends on an insult that affects brain growth, a dynamic process that may take several weeks to become apparent. An ultrasound close to the time of insult may not show any findings, as was reported in a recent case (Mlakar et al., 2016). Follow-up ultrasound and/or postnatal follow-ups may be required. Counseling pregnant women regarding the prognosis, particularly in the less severe forms of microcephaly, may also be challenging.

The available information regarding the spectrum of disease and prognosis of fetuses and newborns exposed to ZIKV during gestation is limited and based on conditions that have other associated morbidities. It appears that congenital ZIKV infection primarily affects the brain tissues during fetal development (Martines et al., 2016; Mlakar et al., 2016), raising speculations that the virus may be neurotropic. However, congenital ZIKV infection has also been found to be associated with ophthalmological lesions in newborns (Freitas et al., 2016) and placenta (Martines et al., 2016; Mlakar et al., 2016). At present, information regarding the long-term prognosis of fetal Zika infection is currently not available.

\subsection{Guillain-Barré syndrome}

While ZIKV infection of children and adults generally produces an inapparent or benign, self-limited illness with mild clinical manifestations, a cluster of Guillain-Barré syndrome (GBS) cases was identified during the French Polynesia outbreak of 2013 (CaoLormeau et al., 2014), of which 42 were associated with ZIKV infection (Musso et al., 2014; Oehler et al., 2014). A further increase in the incidence of GBS was identified in several American countries where ZIKV epidemics have spread since 2015 (WHO. Situation Report. 19 Feb 2016; http://apps.who.int/iris/bitstream/10665/ 204454/1/zikasitrep_19Feb2016_eng.pdf?ua=1). GBS is an autoimmune polyradiculoneuropathy, usually triggered by preceding viral or bacterial infections $2-8$ weeks earlier. Clinically, GBS manifests as progressive weakness, which initiates in the lower limbs and ascends proximally over a few weeks. Accompanying the motor dysfunction and paralysis, patients typically present with reduction or absence of deep tendon reflexes and may also develop cranial nerve disorders.

The spatiotemporal relation between ZIKV outbreaks and an increase in GBS cases was already a strong indicator for a true link between them, but more definitive evidence for this association came from a recently published case-control study performed in French Polynesia (Cao-Lormeau et al., 2016). During this investigation, 42 GBS cases were compared to 98 age-, sex- and residencematched controls. The presence of anti-ZIKV IgM or IgG was observed in 41 (98\%) of GBS cases and in 35 (36\%) controls (Odds Ratio: 59.7, $P<0.0001$ ). Moreover, neutralizing antibodies against ZIKV were found in all (100\%) GBS cases compared with 54 (56\%) controls (odds ratio: 34.1, $p<0.0001$ ). This study also estimated the risk of GBS to be 2.4 per 10,000 ZIKV infections. 
GBS has also been implicated in other arboviral infections, such as DENV (Garg et al., 2015; Simon et al., 2016) and CHIKV (Wielanek et al., 2007), but is believed to be a rare event. The onset of GBS presumably involves an autoimmune process (Yuki and Hartung, 2012), but the underlying factors that influence the association of GBS and ZIKV infection have not yet been determined. It has been suggested that sequential arbovirus infections may exacerbate the immune response and trigger an immunopathogenic process attacking peripheral nerves, thus leading to the onset of GBS (Oehler et al., 2014). So far, ZIKV-induced GBS has been transient in duration and the majority of patients fully recover.

\section{Established and potential ZIKV vectors}

ZIKV circulation has been documented in two ecologically and evolutionarily distinct transmission cycles: an enzootic sylvan cycle, where the virus circulates between arboreal Aedes spp. mosquitoes and non-human primates; and a human cycle, between humans and peridomestic/domestic $A$. spp. mosquitoes. Prior to 2007 our knowledge derived almost exclusively from the enzootic transmission cycle. The first ZIKV isolation was from A. africanus mosquitoes in Uganda (Dick et al., 1952; Haddow et al., 1964), and subsequently in Central African Republic (Berthet et al., 2014) (CAR) and Senegal (Diallo et al., 2014), solidifying its role as a principal vector in the enzootic cycle. ZIKV has been also isolated from Aedes opok in the CAR (Berthet et al., 2014), Aedes apicoargenteus in Uganda, (McCrae and Kirya, 1982) Aedes luteocephalus in Nigeria (Fagbami, 1979) and Senegal (Cornet et al., 1979b) and Aedes vittatus, Aedes furcifer, and A. aegypti formosus in Côte d'Ivoire (AkouaKoffi et al., 2001) and Senegal (Diallo et al., 2014). The virus has also been isolated from other Aedes (Aedes dalzieli, Aedes hirsutus, Aedes unilineatus, Aedes metallicus), and other mosquitoes such as, Anopheles coustani and Mansonia uniformis, that inhabit various enzootic ecotypes (e.g. savannah, forest ground), and curiously from Culex perfuscus, an observation that would require further verification (Diallo et al., 2014). Importantly, the isolation of ZIKV from $A$. vittatus mosquitoes collected within a village supports its putative role as a bridge vector of enzootic ZIKV strains into agricultural villages at the zone of emergence, and potentially into the human transmission cycle (Diallo et al., 2014). Collectively, these mosquitoes belong mainly to arboreal or rural species of the genus Aedes, and more precisely to the Aedimorphus, Diceromyia and Stegomyia subgenera.

While transmission of ZIKV is assumed to occur mainly in urban settings via the anthropophilic A. aegypti mosquito, as evidenced by limited field surveillance (Marchette et al., 1969; Olson et al., 1981) and experimental studies (Boorman and Porterfield, 1956; Cornet et al., 1979a; Li et al., 2012), A. hensilli (Ledermann et al., 2014) and/or A. polynesiensis (Musso et al., 2014) may serve as vectors in niche ecotypes. In 2007 in Gabon, urban ZIKV transmission was associated with A. albopictus (Grard et al., 2014). Further experimental studies (Wong et al., 2013) supported a role for Asian populations of $A$. albopictus as vectors of ZIKV transmission. Given its invasive nature and extensive geographic distribution in tropical as well as temperate settings, there is the potential for A. albopictus to become a major ZIKV vector globally. The introduction of ZIKV and the explosive epidemic under way in the Americas beg the question of its potential role in ZIKV transmission in urban and rural settings. Given that $A$. albopictus has been implicated in Asia as the bridge vector for sylvatic DENV into the urban cycle [reviewed in (Hanley et al., 2013; Vasilakis et al., 2011)], would this vector serve as the bridge to facilitate reverse spillback and establishment of an enzootic ZIKV transmission cycle in the Americas? Certainly a number of New World mosquitoes involved in the enzootic transmission of yellow fever virus (YFV), including
Haemagogus albomaculatus, Haemagogus spegazzini, Haemagogus janthinomys, Sabethes chloropterus, Sabethes albipivus, Sabethes glaucodaemon, Sabethes soperi, and Sabethes cyaneus, Psorophora ferox and Aedes serratus [reviewed in (Hanley et al., 2013)] could serve as competent vectors of ZIKV and should be evaluated experimentally.

\section{Potential mechanisms of Zika epidemic emergence since 2013}

The sudden and dramatic emergence of ZIKV into a humanmosquito-human transmission cycle to generate major outbreaks since 2007 begs the question of what precipitated these events. There are several hypotheses to explain this emergence; the following is a plausible but not an exhaustive set of examples:

\subsection{Adaptive evolution for mosquito transmission}

Sometime during the past decade ZIKV underwent adaptive evolution (or genetic drift resulting from a population bottleneck and not the result of selection) that resulted in more efficient transmission by A. aegypti and perhaps other closely related mosquito vectors (in the Aedes (Stegomyia) subgenus) such as Aedes hensilii incriminated in Yap (Ledermann et al., 2014) or A. polynesiensis suspected as a vector in French Polynesia (Musso et al., 2014). Phylogenetic analyses (see above) suggest that this adaptive evolution would have occurred in Southeast Asia or the South Pacific. This hypothesis is supported by the established presence of ZIKV in Southeast Asia since at least 1966, including its isolation from A. aegypti in Malaysia (Marchette et al., 1969), yet the lack of evidence for major urban epidemics. A precedent for this explanation is the vector-adaptive evolution of the Indian Ocean Lineage of chikungunya virus for enhanced transmission by A. albopictus, which has been documented through a series of mutations in the envelope glycoprotein genes that apparently enhance infection of the midgut epithelial cells (Tsetsarkin et al., 2014). Comparisons of the infectivity of older Asian lineage ZIKV strains with recent isolates, followed by reverse genetic approaches to test the effects of recent mutations, can be used to test this hypothesis.

\subsection{Adaptive evolution for human viremia}

Recently the Asian ZIKV lineage may have adapted to generate higher viremia levels in humans, which would lead to more efficient mosquito infection and higher levels of transmission and spread. Higher viremia could also enhance transplacental transmission to explain the emergence of microcephaly, or changes in cell tropism could be involved. Informatic studies of ZIKV sequences, which suggest an increase in the virus' use of human codons, supports this hypothesis (Faye et al., 2014). However, the potential link between human codon usage and enhanced human infection will require studies with human cells and/or animal models. Ultimately, this hypothesis will be difficult to test because even nonhuman primates (NHPs) may not respond to ZIKV infection in exactly the same manner as humans.

\subsection{Herd immunity from endemic exposure in Africa and Asia limits outbreak magnitude}

Enzootic and/or endemic ZIKV circulation in Asia results in relatively stable levels of human herd immunity that limit the potential for recognized outbreaks. Limited human seroprevalence in a few Asian sites supports this hypothesis, and recent studies of longitudinal population immunity to CHIKV in the Philippines 
suggest cyclic endemic circulations that maintains herd immunity in the range of approximately $20-50 \%$, levels that may reduce the risk of major epidemics (Yoon et al., 2015). Enhanced surveillance in Asia is needed to assess levels of ZIKV transmission and seroprevalence, and logistically difficult studies to determine if an enzootic cycle exists in NHPs or other vertebrates are also needed. Stable endemic ZIKV circulation could also explain the lack of recognized human infections because most are clinically indistinguishable from DENV infections and ZIKV diagnostics are not readily available. If herd immunity reaches high levels by the time women reach child-bearing age, then microcephaly could occur at lower incidence than in epidemic regions of the Americas; continuous, low incidence of microcephaly could also go unrecognized in an endemic region where there are not enough cases to raise suspicion of a cause other than historically-recognized etiologies such as cytomegalovirus, herpesviruses, Rubella, toxoplasmosis, or toxic exposures.

\subsection{Stochastic introductions into the Pacific and Americas}

The recent ZIKV emergence resulted from the chance introduction of the virus into naïve populations in the South Pacific, where competent vectors mediated sufficient amplification to raise the risk of transport to the Americas, also occupied by a completely ZIKV-naïve population. Increased air travel undoubtedly has increased the risk of such introductions during recent decades, and athletic competitions in Brazil are believed to have brought travelers from the South Pacific around the time that ZIKV circulation was discovered there (Musso, 2015; Petersen et al., 2016).

\section{Prospects for control of Zika virus spread and disease}

\subsection{Vaccines}

A number of approaches have successfully led to efficacious flavivirus vaccines. Clinically approved vaccines are available for four flaviviruses. (i) Vaccine for yellow fever virus (17D strain) is a live attenuated virus that has been successfully used in humans since 1937. (ii) Both inactivated-virus vaccine and live attenuated vaccine (14-14-2 strain) are available for Japanese encephalitis virus. (iii) An inactivated-virus vaccine is used for tick-borne encephalitis virus. (iv) A chimeric virus, representing the 17D vaccine strain of yellow fever virus that contains the structural membrane and envelope genes from dengue virus, was recently approved for clinical use for dengue virus. The above approaches could be adopted for ZIKV vaccine development. In addition, subunit vaccines representing ZIKV proteins, DNA vaccines expressing viral proteins, and other viral vectors expressing viral antigens could be explored. It should be noted that each vaccine approach has its pros and cons. For example, compared with a live attenuated virus vaccine, subunit vaccines could have better safety and shorter development time; reversely, a live attenuated virus vaccine may trigger more robust humoral and cellular immune response for better protection. Therefore, complementary approaches should be explored simultaneously to advance vaccine for ZIKV.

\subsection{Therapeutics}

No clinically approved therapy is currently available for treatment of any flavivirus infections (Lim et al., 2013). Over the past decade, significant effort has been made towards dengue drug discovery. Due to the similarity between ZIKV and dengue virus, knowledge derived from dengue drug discovery could be applied to ZIKV; it is not unreasonable to speculate that inhibitors active against both ZIKV and dengue virus could be found. However, cautions should be taken when extrapolating dengue experience to ZIKV drug discovery. This is because the biology of the two viruses could be very different. Understanding the disease biology is essential for therapeutics development for any pathogens. For example, it is important to know where ZIKV replicates in patients during the course of infection and disease; this information will guide drug discovery in terms of where the inhibitors should be distributed to during treatment. The duration of viremia in patients determines the therapeutic window. Ideally, to prevent $\mathrm{MC}$ in a ZIKV-infected pregnant woman, the inhibitor would need to possess a pharmacological property of brain penetration and to block viral replication in the fetal brain; meanwhile, the compound would also need to provide a systemic exposure high enough to inhibit viral replication in various other organs; therefore, an ideal drug needs to pharmacologically inhibit ZIKV in both brain and systemic sites. However, a non-brain penetrating compound with good systemic exposure may restrict viremia and prevent fetus (including fetal brain) infection. Animal models that recapture human diseases of ZIKV infection, including MC and GBS, are urgently needed for vaccine and therapeutic development. Because pregnant women comprise major target populations for ZIKV therapy, this poses a major challenge and will take extra time for clinical development. Once a drug becomes available, the compound could be used for prophylaxis for travelers, family members of a household with infected individual(s), and the general population during an epidemic.

\subsection{Vector control}

In the immediate future, while vaccines and therapeutics remain unavailable probably for at least a few years, the best prospects for controlling Zika virus rely on reducing contact between the vector, probably $A$. aegypti in most regions, and susceptible humans, especially pregnant women who represent the highest risk of severe disease in the form of fetal MC. The most effective approach to reducing such contact would be to eliminate or reduce these mosquito population, a strategy that succeeded in much of Latin America during the second half of the 20th century. However, this eradication campaign relied heavily on the use of DDT and other persistent, highly toxic insecticides to kill adult female mosquitoes, methods that are considered by many to be environmentally unacceptable today (Gubler, 1987, 1997). Governments also imposed major penalties for residents who failed to eliminate sources of standing water on their properties where A. aegypti lay their eggs for larval development. With these measures weakened and rapidly growing neotropical cities now fully reinfested with $A$. aegypti, the prospects for renewed eradication are daunting (Gubler, 2011).

Reductions in A. aegypti populations could theoretically be achieved using one or more of several approaches: 1) Elimination or protection of water containers ranging from large tanks used to store household water where a reliable municipal supply is not available to used tires and other refuse that fill with rainwater and serve as larval habitats. This approach generally relies on community engagement and personal responsibility, sometimes supplemented by penalties for allowing larval development to occur on ones property (Winch et al., 2002). However, source reduction has achieved only modest success in controlling dengue because one or a few properties in a given neighborhood can produce sufficient mosquitoes to sustain transmission. Larvicides can also be applied to these sources but, again, partial treatment can allow sufficient vector populations for transmission to persist. Killing the adult female A. aegypti using traditional fogging with insecticide aerosols is generally not effective unless implemented within homes or other places where people are exposed to virus infection because 
these vectors tend to stay indoors where insecticides applied from trucks or airplanes are unlikely to penetrate. Indoor spraying including the application of residual insecticides that may also have repellant activity can be more effective but his expensive and laborintensive (Fernandez-Salas et al., 2015; Paredes-Esquivel et al., 2016).

Several new technologies show promise to control A. aegypti. One involves the release of genetically modified male mosquitoes that express a dominant, lethal gene at the larval stage, resulting in the death of all offspring from mating with wild females, leaving no risk for persistence of the transgene in nature. This approach has had dramatic effects on reducing populations of $A$. aegypti when applied on a small scale (Wise de Valdez et al., 2011), but the logistical, technical and financial challenges of scaling up this method to impact the huge tropical cities where Zika and other arboviruses circulate have not been addressed.

Another approach to reducing the transmission of dengue and potentially Zika virus is to release A. aegypti infected with endosymbiotic Wolbachia bacteria, which can spread through natural populations and suppress viral transmission by interfering with replication in the mosquito. Controlled releases have resulted in successful spread and now releases into dengue-endemic tropical locations are being implemented to look for evidence that disease incidence can be reduced (Ritchie et al., 2015). A potential limitation of this approach is the need to release Wolbachia-infected mosquitoes over wide geographic ranges to overcome the limited A. aegypti flight range, and the possibility that arboviruses will rapidly evolve mechanisms to overcome the inhibitory effects of the Wolbachia.

Finally, an approach that might represent a near-term way to reduce $A$. aegypti populations is the use of lethal traps, which have been designed to be inexpensive and relatively maintenance-free. An autocidal gravid ovitrap has been shown to reduce $A$. aegypti populations under field conditions in two isolated urban areas of Puerto Rico by $53-70 \%$ using 3-4 traps per home in $81 \%$ of houses (Barrera et al., 2014). Many other designs are being tested, and these kinds of simple traps may be very useful when combined with source reduction and adulticide applications in regions at risk for Zika virus transmission by A. aegypti.

\section{Critical gaps and needs}

With ZIKV spreading rapidly throughout the topical and subtropical Americas, critical gaps remain in our knowledge of this virus yet rapid responses to contain the outbreak must be implemented immediately. The causative connection between ZIKV infection and MC as well as GBS needs to be confirmed by welldesigned case-control and prospective cohort studies to ensure that another etiology or co-factor is not overlooked. Assuming that the causal relationship is confirmed, in the near term a reduction in contact between people and $A$. aegypti through the use of repellents, insecticide-impregnated clothing, and simple measures to prevent these mosquitoes from entering homes and places of work has the best chance of reducing ZIKV transmission and spread. Pregnant women need to be especially well educated and vigilant to avoid mosquito bites. Although source reduction to eliminate vector larval habitats has had limited success in controlling dengue, perhaps the widespread media coverage of microcephaly cases that evokes a major emotional response will motivate at-risk populations to take these efforts more seriously and to also use all protective measures available to reduce contact with vectors. Regardless, source reduction will likely need to be supplemented by methods to control adult female mosquitoes, including indoor insecticide applications. Additional surveillance combined with experimental infections are needed to confirm the assumption that
A. aegypti is the main ZIKV vector, or to also direct control measures to other mosquitoes involved such as $A$. albopictus and perhaps others.

Beyond the immediate future, past work on flavivirus vaccines and therapeutics should provide platforms and approaches that can accelerate product development for ZIKV. However, animal models need to be developed quickly to begin testing some products that may be developed by the end of this year. In the longer-term, better surveillance facilitated by inexpensive, simple point-of-care diagnostics will be needed to identify sites with sufficient disease incidence to conduct clinical efficacy trials on promising products during the coming years. This may be especially challenging once the current epidemics subside with growing herd immunity and diagnostics become critical to distinguish ZIKV infections from DENV and CHIKV, as well as other causes of acute, nonspecific febrile illness. Overcoming the major challenge of cross-reactive serodiagnostics in dengue-hyperendemic locations could greatly enhance identification of regions still at risk of major ZIKV outbreaks, which would be ideal sites for clinical trials and other interventions such as novel vector control methods. Finally, surveillance for arboviral disease also needs to be strengthened in Africa and Asia, where the potential for major outbreaks remains unknown 69 years after the discovery of ZIKV.

\section{Acknowledgments}

The authors' research on Zika virus is supported by NIH grants AI120942 and AI093491 to SCW, and AI121207 to AIK.

\section{References}

Akoua-Koffi, C., Diarrassouba, S., Benie, V.B., Ngbichi, J.M., Bozoua, T., Bosson, A. Akran, V., Carnevale, P., Ehouman, A., 2001. Investigation surrounding a fata case of yellow fever in Cote d'Ivoire in 1999. Bull. Soc. Pathol. Exot. 94, 227-230.

Atkinson, B., Hearn, P., Afrough, B., Lumley, S., Carter, D., Aarons, E.J., 2016. Detection of Zika virus in semen. Emerg. Infect. Dis. (in press).

Barrera, R. Amador, M., Acevedo, V., Caban, B., Felix, G., Mackay, A.J., 2014. Use of the CDC autocidal gravid ovitrap to control and prevent outbreaks of Aedes aegypti (Diptera: Culicidae). J. Med. Entomol. 51, 145-154.

Barrows, N.J., Jamison, S.F., Bradrick, S.S., Le Sommer, C., Kim, S.Y., Pearson, J., GarciaBlanco, M.A., 2014. Functional genomics approach for the identification of human host factors supporting dengue viral propagation. Methods Mol. Biol. 1138 285-299.

Bearcroft, W.G., 1956. Zika virus infection experimentally induced in a human volunteer. Trans. R. Soc. Trop. Med. Hyg. 50, 442-448.

Berthet, N., Nakoune, E., Kamgang, B., Selekon, B., Descorps-Declere, S., Gessain, A. Manuguerra, J.C., Kazanji, M., 2014. Molecular characterization of three Zika flaviviruses obtained from sylvatic mosquitoes in the Central African Republic. Vector Borne Zoonotic Dis. 14, 862-865.

Bidet, K., Garcia-Blanco, M.A., 2014. Flaviviral RNAs: weapons and targets in the war between virus and host. Biochem. J. 462, 215-230.

Boorman, J.P., Porterfield, J.S., 1956. A simple technique for infection of mosquitoes with viruses; transmission of Zika virus. Trans. R. Soc. Trop. Med. Hyg. 50 $238-242$.

Brasil, P., Pereira Jr., J.P., Raja Gabaglia, C., Damasceno, L., Wakimoto, M., Ribeiro Nogueira, R.M., Carvalho de Sequeira, P., Machado Siqueira, A., Abreu de Carvalho, L.M., Cotrim da Cunha, D., Calvet, G.A., Neves, E.S., Moreira, M.E. Rodrigues Baiao, A.E., Nassar de Carvalho, P.R., Janzen, C., Valderramos, S.G. Cherry, J.D., Bispo de Filippis, A.M., Nielsen-Saines, K., 2016. Zika virus infection in pregnant women in Rio de Janeiro - preliminary report. N. Engl. J. Med.

Calvet, G., Aguiar, R.S., Melo, A.S., Sampaio, S.A., de Filippis, I., Fabri, A., Araujo, E.S. de Sequeira, P.C., de Mendonca, M.C., de Oliveira, L., Tschoeke, D.A. Schrago, C.G., Thompson, F.L., Brasil, P., Dos Santos, F.B., Nogueira, R.M., Tanuri, A., de Filippis, A.M., 2016. Detection and sequencing of Zika virus from amniotic fluid of fetuses with microcephaly in Brazil: a case study. Lancet Infect. Dis.

Campos, G.S., Bandeira, A.C., Sardi, S.I., 2015. Zika virus outbreak, Bahia, Brazil. Emerg. Infect. Dis. 21, 1885-1886.

Cao-Lormeau, V.M., Roche, C., Teissier, A., Robin, E., Berry, A.L., Mallet, H.P., Sall, A.A. Musso, D., 2014. Zika virus, French polynesia, South pacific, 2013. Emerg. Infect. Dis. 20, 1085-1086.

Cao-Lormeau, V.M., Blake, A., Mons, S., Lastere, S., Roche, C., Vanhomwegen, J. Dub, T., Baudouin, L., Teissier, A., Larre, P., Vial, A.L., Decam, C., Choumet, V. Halstead, S.K., Willison, H.J., Musset, L., Manuguerra, J.C., Despres, P. Fournier, E., Mallet, H.P., Musso, D., Fontanet, A., Neil, J., Ghawche, F., 2016 
Guillain-Barre syndrome outbreak associated with Zika virus infection in French Polynesia: a case-control study. Lancet.

Chen, R., Vasilakis, N., 2011. Dengue-quo tu et quo vadis? Viruses 3, 1562-1608.

Committee, S.P., 2016. SMFM statement: ultrasound screening for fetal microcephaly following Zika virus exposure. Am. J. Obstet. Gynecol.

Cornet, M., Robin, Y., Adam, C., Valade, M., Calvo, M., 1979a. Comparison between experimental transmission of yellow fever and Zika viruses in Aedes aegypti. Cah. ORSTOM Ser. Ent. Med. Parasitol. 17, 47-53.

Cornet, M., Robin, Y., Chateau, R., Heme, G., Adam, C., 1979b. Isolement d'arbovirus au Senegal oriental a partir de moustiques (1972-1977) et note surl'epidemiologie des virus transmis par les Aedes, en particulier du virus amaril ORSTOM Entomol. Med. Parasitol. 17, 149-163.

Costa, F., Sarno, M., Khouri, R., de Paulo Freitas, B., Siqueira, I., Ribeiro, G.S., Ribeiro, H.C., Campos, G.S., Alcantara, L.C., Reis, M.G., Weaver, S.C., Vasilakis, N. Ko, A.I., Almeida, A.R., 2016. Emergence of congenital Zika syndrome: viewpoint from the front lines. Ann. Intern. Med. (in press).

Daffis, S., Szretter, K.J., Schriewer, J., Li, J., Youn, S., Errett, J., Lin, T.Y., Schneller, S. Zust, R., Dong, H., Thiel, V., Sen, G.C., Fensterl, V., Klimstra, W.B., Pierson, T.C. Buller, R.M., Gale Jr., M., Shi, P.Y., Diamond, M.S., 2010. 2'-O methylation of the viral mRNA cap evades host restriction by IFIT family members. Nature 468, $452-456$.

Diallo, D., Sall, A.A., Diagne, C.T., Faye, O., Faye, O., Ba, Y., Hanley, K.A. Buenemann, M., Weaver, S.C., Diallo, M., 2014. Zika virus emergence in mosquitoes in southeastern Senegal, 2011. Plos One 9, e109442.

Dick, G.W., Kitchen, S.F., Haddow, A.J., 1952. Zika virus. I. Isolations and serological specificity. Trans. R. Soc. Trop. Med. Hyg. 46, 509-520.

Duffy, M.R., Chen, T.H., Hancock, W.T., Powers, A.M., Kool, J.L., Lanciotti, R.S., Pretrick, M., Marfel, M., Holzbauer, S., Dubray, C., Guillaumot, L., Griggs, A., Bel, M., Lambert, A.J., Laven, J., Kosoy, O., Panella, A., Biggerstaff, B.J., Fischer, M., Hayes, E.B., 2009. Zika virus outbreak on Yap Island, Federated States of Micronesia. N. Engl. J. Med. 360, 2536-2543.

Enfissi, A., Codrington, J., Roosblad, J., Kazanji, M., Rousset, D., 2016. Zika virus genome from the Americas. Lancet 387, 227-228.

Fagbami, A.H., 1979. Zika virus infections in Nigeria: virological and seroepidemiological investigations in Oyo State. J. Hyg. Lond. 83, 213-219.

Faria, N.R., Azevedo, R.D., Kraemer, M.U., Souza, R., Cunha, M.S., Hill, S.C., Theze, J., Bonsall, M.B., Bowden, T.A., Rissanen, I., Rocco, I.M., Nogueira, J.S., Maeda, A.Y., Vasami, F.G., Macedo, F.L., Suzuki, A., Rodrigues, S.G., Cruz, A.C., Nunes, B.T., Medeiros, D.B., Rodrigues, D.S., Nunes Queiroz, A.L., Silva, E.V., Henriques, D.F., Travassos da Rosa, E.S., de Oliveira, C.S., Martins, L.C., Vasconcelos, H.B. Casseb, L.M., Simith, D.B., Messina, J.P., Abade, L., Lourenco, J., Alcantara, L.C. Lima, M.M., Giovanetti, M., Hay, S.I., de Oliveira, R.S., Lemos, P.D., Oliveira, L.F., de Lima, C.P., da Silva, S.P., Vasconcelos, J.M., Franco, L., Cardoso, J.F., Vianez Junior, J.L., Mir, D., Bello, G., Delatorre, E., Khan, K., Creatore, M., Coelho, G.E., de Oliveira, W.K., Tesh, R., Pybus, O.G., Nunes, M.R., Vasconcelos, P.F., 2016. Zika virus in the Americas: early epidemiological and genetic findings. Science online.

Faye, O., Freire, C.C., Iamarino, A., Faye, O., de Oliveira, J.V., Diallo, M., Zanotto, P.M. Sall, A.A., 2014. Molecular evolution of Zika virus during its emergence in the 20(th) century. Plos Negl. Trop. Dis. 8, e2636.

Fernandez-Salas, I., Danis-Lozano, R., Casas-Martinez, M., Ulloa, A., Bond, J.G. Marina, C.F., Lopez-Ordonez, T., Elizondo-Quiroga, A., Torres-Monzon, J.A., DiazGonzalez, E.E., 2015. Historical inability to control Aedes aegypti as a main contributor of fast dispersal of chikungunya outbreaks in Latin America. Antivir Res. 124, 30-42.

Freitas, B.P., Dias, J.R.O., Prazeres, J., Sacramento, G.A., Ko, A.I., Maia, M., Belfort Jr., R. 2016. Ocular Findings in Infants With Microcephaly Associated With Presumed Zika Virus Congenital Infection in Salvador. JAMA Ophthalmol, Brazil.

Garcia-Blanco, M.A., 2015. Know thyself. RNA 21, 525-526.

Garg, R.K., Malhotra, H.S., Jain, A., Malhotra, K.P., 2015. Dengue-associated neuromuscular complications. Neurol. India 63, 497-516.

Grard, G., Caron, M., Mombo, I.M., Nkoghe, D., Mboui Ondo, S., Jiolle, D., Fontenille, D., Paupy, C., Leroy, E.M., 2014. Zika virus in Gabon (Central Africa)2007: a new threat from Aedes albopictus? Plos Negl. Trop. Dis. 8, e2681.

Gubler, D.J., 1987. Dengue and dengue hemorrhagic fever in the Americas. P. R. Health Sci. J. 6, 107-111.

Gubler, D.J., 1997. Dengue and dengue hemorrhagic fever: its history and resurgence as a global public health problem. In: Gubler, D.J., Kuno, G. (Eds.), Dengue and Dengue Hemorrhagic Fever. CAB International, New York, pp. 1-22.

Gubler, D.J., 2011. Dengue, urbanization and globalization: the unholy trinity of the 21(st) century. Trop. Med. Health 39, 3-11.

Haddow, A.J., Williams, M.C., Woodall, J.P., Simpson, D.I., Goma, L.K., 1964. Twelve isolations of Zika virus from Aedes (Stegomyia) africanus (Theobald) taken in and above a Uganda forest. Bull. World Health Org. 31, 57-69.

Hamel, R., Dejarnac, O., Wichit, S., Ekchariyawat, P., Neyret, A., Luplertlop, N., Perera-Lecoin, M., Surasombatpattana, P., Talignani, L., Thomas, F., CaoLormeau, V.M., Choumet, V., Briant, L., Despres, P., Amara, A., Yssel, H., Misse, D., 2015. Biology of Zika virus infection in human skin cells. J. Virol. 89, 8880-8896.

Hanley, K.A., Monath, T.P., Weaver, S.C., Rossi, S.L., Richman, R.L., Vasilakis, N., 2013. Fever versus fever: the role of host and vector susceptibility and interspecific competition in shaping the current and future distributions of the sylvatic cycles of dengue virus and yellow fever virus. Infect. Genet. Evol.

Heang, V., Yasuda, C.Y., Sovann, L., Haddow, A.D., Travassos da Rosa, A.P., Tesh, R.B. Kasper, M.R., 2012. Zika virus infection, Cambodia, 2010. Emerg. Infect. Dis. 18, $349-351$.
Horwood, P., Bande, G., Dagina, R., Guillaumot, L., Aaskov, J., Pavlin, B., 2013. The threat of chikungunya in Oceania. West. Pac. Surveill. Response J. 4, 8-10.

Krishnan, M.N., Ng, A., Sukumaran, B., Gilfoy, F.D., Uchil, P.D., Sultana, H., Brass, A.L. Adametz, R., Tsui, M., Qian, F., Montgomery, R.R., Lev, S., Mason, P.W., Koski, R.A., Elledge, S.J., Xavier, R.J., Agaisse, H., Fikrig, E., 2008. RNA interference screen for human genes associated with West Nile virus infection. Nature 455, 242-245.

Lanciotti, R.S., Kosoy, O.L., Laven, J.J., Velez, J.O., Lambert, A.J., Johnson, A.J., Stanfield, S.M., Duffy, M.R., 2008. Genetic and serologic properties of Zika virus associated with an epidemic, Yap State, Micronesia, 2007. Emerg. Infect. Dis. 14, 1232-1239.

Le Sommer, C., Barrows, N.J., Bradrick, S.S., Pearson, J.L., Garcia-Blanco, M.A., 2012. $G$ protein-coupled receptor kinase 2 promotes flaviviridae entry and replication. Plos Negl. Trop. Dis. 6, e1820.

Ledermann, J.P., Guillaumot, L., Yug, L., Saweyog, S.C., Tided, M., Machieng, P., Pretrick, M., Marfel, M., Griggs, A., Bel, M., Duffy, M.R., Hancock, W.T., HoChen, T., Powers, A.M., 2014. Aedes hensilli as a potential vector of Chikungunya and Zika viruses. Plos Negl. Trop. Dis. 8, e3188.

Li, M.I., Wong, P.S., Ng, L.C., Tan, C.H., 2012. Oral susceptibility of Singapore Aedes (Stegomyia) aegypti (Linnaeus) to Zika virus. Plos Negl. Trop. Dis. 6, e1792.

Lim, S.P., Wang, Q.Y., Noble, C.G., Chen, Y.L., Dong, H., Zou, B., Yokokawa, F., Nilar, S. Smith, P., Beer, D., Lescar, J., Shi, P.Y., 2013. Ten years of dengue drug discovery: progress and prospects. Antivir. Res. 100, 500-519.

Ma, H., Dang, Y., Wu, Y., Jia, G., Anaya, E., Zhang, J., Abraham, S., Choi, J.G., Shi, G., Qi, L., Manjunath, N., Wu, H., 2015. A CRISPR-based screen identifies genes essential for West-Nile-virus-induced cell death. Cell Rep. 12, 673-683.

Macnamara, F.N., 1954. Zika virus: a report on three cases of human infection during an epidemic of jaundice in Nigeria. Trans. R. Soc. Trop. Med. Hyg. 48, 139-145.

Manokaran, G., Finol, E., Wang, C., Gunaratne, J., Bahl, J., Ong, E.Z., Tan, H.C., Sessions, O.M., Ward, A.M., Gubler, D.J., Harris, E., Garcia-Blanco, M.A., Ooi, E.E., 2015. Dengue subgenomic RNA binds TRIM25 to inhibit interferon expression for epidemiological fitness. Science 350, 217-221.

Marchette, N.J., Garcia, R., Rudnick, A., 1969. Isolation of Zika virus from Aedes aegypti mosquitoes in Malaysia. Am. J. Trop. Med. Hyg. 18, 411-415.

Marrs, C., Olson, G., Saade, G., Hankins, G., Wen, T., Patel, J., Weaver, S., 2016. Zika virus and pregnancy: a review of the literature and clinical considerations. Am. J. Perinatol.

Martines, R.B., Bhatnagar, J., Keating, M.K., Silva-Flannery, L., Muehlenbachs, A., Gary, J., Goldsmith, C., Hale, G., Ritter, J., Rollin, D., Shieh, W.J., Luz, K.G., Ramos, A.M., Davi, H.P., Kleber de Oliveria, W., Lanciotti, R., Lambert, A., Zaki, S., 2016. Notes from the field: evidence of Zika virus infection in brain and placental tissues from two congenitally infected newborns and two fetal losses - Brazil, 2015. MMWR Morb. Mortal. Wkly. Rep. 65, 159-160.

McCrae, A.W., Kirya, B.G., 1982. Yellow fever and Zika virus epizootics and enzootics in Uganda. Trans. R. Soc. Trop. Med. Hyg. 76, 552-562.

McGee, C.E., Tsetsarkin, K.A., Guy, B., Lang, J., Plante, K., Vanlandingham, D.L., Higgs, S., 2011. Stability of yellow fever virus under recombinatory pressure as compared with chikungunya virus. Plos One 6, e23247.

Mlakar, J., Korva, M., Tul, N., Popovic, M., Poljsak-Prijatelj, M., Mraz, J., Kolenc, M., Resman Rus, K., Vesnaver Vipotnik, T., Fabjan Vodusek, V., Vizjak, A., Pizem, J., Petrovec, M., Avsic Zupanc, T., 2016. Zika virus associated with microcephaly. N. Engl. J. Med.

Musso, D., 2015. Zika virus transmission from French Polynesia to Brazil. Emerg. Infect. Dis. 21, 1887.

Musso, D., Nilles, E.J., Cao-Lormeau, V.M., 2014. Rapid spread of emerging Zika virus in the Pacific area. Clin. Microbiol. Infect. 20, 0595-596.

Oehler, E., Watrin, L., Larre, P., Leparc-Goffart, I., Lastere, S., Valour, F., Baudouin, L., Mallet, H., Musso, D., Ghawche, F., 2014. Zika virus infection complicated by Guillain-Barre syndrome-case report, French Polynesia, December 2013. Euro Surveill 19.

Olson, J.G., Ksiazek, T.G., Suhandiman, Triwibowo, 1981. Zika virus, a cause of fever in Central Java, Indonesia. Trans. R. Soc. Trop. Med. Hyg. 75, 389-393.

Paredes-Esquivel, C., Lenhart, A., Del Rio, R., Leza, M.M., Estrugo, M., Chalco, E., Casanova, W., Miranda, M.A., 2016. The impact of indoor residual spraying of deltamethrin on dengue vector populations in the Peruvian Amazon. Acta Trop. $154,139-144$

Passemard, S., Kaindl, A.M., Verloes, A., 2013. Microcephaly. Handb. Clin. Neurol. 111, $129-141$.

Petersen, E., Wilson, M.E., Touch, S., McCloskey, B., Mwaba, P., Bates, M., Dar, O., Mattes, F. Kidd, M. Ippolito, G. Azhar, E.I. Zumla, A., 2016. Unexpected and rapid spread of Zika virus in the Americas - implications for public health preparedness for mass gatherings at the 2016 Brazil Olympic Games. Int. J. Infect. Dis. IJID Off. Publ. Int. Soc. Infect. Dis.

Ritchie, S.A., Townsend, M., Paton, C.J., Callahan, A.G., Hoffmann, A.A., 2015. Application of wMelPop Wolbachia strain to crash local populations of Aedes aegypti. Plos Negl. Trop. Dis. 9, e0003930.

Sarno, M. Sacramento, G.A., Khouri, R., do Rosario, M.S., Costa, F., Archanjo, G. Santos, L.A., Nery Jr., N., Vasilakis, N., Ko, A.I., de Almeida, A.R., 2016. Zika virus infection and stillbirths: a case of hydrops fetalis, hydranencephaly and fetal demise. Plos Negl. Trop. Dis. 10, e0004517.

Sessions, O.M., Barrows, N.J., Souza-Neto, J.A., Robinson, T.J., Hershey, C.L. Rodgers, M.A., Ramirez, J.L., Dimopoulos, G., Yang, P.L., Pearson, J.L., GarciaBlanco, M.A., 2009. Discovery of insect and human dengue virus host factors. Nature 458, 1047-1050.

Simon, O., Billot, S., Guyon, D., Daures, M., Descloux, E., Gourinat, A.C., Molko, N., Dupont-Rouzeyrol, M., 2016. Early Guillain-Barre syndrome associated with 
acute dengue fever. J. Clin. Virol. 77, 29-31.

Tang, H., Hammock, C., Ogden, S.C., Wen, Z., Oian, X., Li, Y., Yao, B., Shin, J., Zhang, F. Lee, E.M., Christian, K.M., Didier, R.A., Jin, P., Song, H., Ming, G., 2016. Zika virus infects human cortical neural progenitors and attenuates their growth. Cell Stem Cell online.

Taucher, C., Berger, A., Mandl, C.W., 2010. A trans-complementing recombination trap demonstrates a low propensity of flaviviruses for intermolecular recombination. J. Virol. 84, 599-611.

Thomas, D.L., Sharp, T.M., Torres, J., Armstrong, P.A., Munoz-Jordan, J., Ryff, K.R. Martinez-Quinones, A., Arias-Berrios, J., Mayshack, M., Garayalde, G.J. Saavedra, S., Luciano, C.A., Valencia-Prado, M., Waterman, S., Rivera-Garcia, B., 2016. Local transmission of Zika virus - Puerto Rico, November 23, 2015-January 28, 2016. MMWR Morb. Mortal. Wkly. Rep. 65, 154-158.

Tsetsarkin, K.A., Chen, R., Yun, R., Rossi, S.L., Plante, K.S., Guerbois, M., Forrester, N., Perng, G.C., Sreekumar, E., Leal, G., Huang, J., Mukhopadhyay, S., Weaver, S.C., 2014. Multi-peaked adaptive landscape for chikungunya virus evolution predicts continued fitness optimization in Aedes albopictus mosquitoes. Nat. Commun. 5, 4084.

Wise de Valdez, M.R., Nimmo, D., Betz, J., Gong, H.F., James, A.A., Alphey, L., Black, W.C.t., 2011. Genetic elimination of dengue vector mosquitoes. Proc. Natl. Acad. Sci. U. S. A. 108, 4772-4775.

Vasilakis, N., Cardosa, J., Hanley, K.A., Holmes, E.C., Weaver, S.C., 2011. Fever from the forest: prospects for the continued emergence of sylvatic dengue virus and its impact on public health. Nat. Rev. Microbiol. 9, 532-541.
Wang, E., Ni, H., Xu, R., Barrett, A.D., Watowich, S.J., Gubler, D.J., Weaver, S.C., 2000 Evolutionary relationships of endemic/epidemic and sylvatic dengue viruses. J. Virol. 74, 3227-3234.

Wielanek, A.C., Monredon, J.D., Amrani, M.E., Roger, J.C., Serveaux, J.P., 2007. Guillain-Barre syndrome complicating a Chikungunya virus infection. Neurology 69, 2105-2107.

Winch, P.J., Leontsini, E., Rigau-Perez, J.G., Ruiz-Perez, M., Clark, G.G., Gubler, D.J. 2002. Community-based dengue prevention programs in Puerto Rico: impact on knowledge, behavior, and residential mosquito infestation. Am. J. Trop. Med. Hyg. 67, 363-370.

Wong, P.S., Li, M.Z., Chong, C.S., Ng, L.C., Tan, C.H., 2013. Aedes (Stegomyia) albopictus (Skuse): a potential vector of Zika virus in Singapore. Plos Negl. Trop. Dis. 7, e2348.

Yoon, I.K., Alera, M.T., Lago, C.B., Tac-An, I.A., Villa, D., Fernandez, S., Thaisomboonsuk, B., Klungthong, C., Levy, J.W., Velasco, J.M., Roque Jr., V.G., Salje, H., Macareo, L.R., Hermann, L.L., Nisalak, A., Srikiatkhachorn, A., 2015 High rate of subclinical chikungunya virus infection and association of neutralizing antibody with protection in a prospective cohort in the Philippines. Plos Negl. Trop. Dis. 9, e0003764.

Yuki, N., Hartung, H.P., 2012. Guillain-Barre syndrome. N. Engl. J. Med. 366, 2294-2304.

Zanluca, C., de Melo, V.C., Mosimann, A.L., Dos Santos, G.I., Dos Santos, C.N., Luz, K. 2015. First report of autochthonous transmission of Zika virus in Brazil. Mem. Inst. Oswaldo Cruz 110, 569-572. 